\title{
Formación académica en información y comunicación: problematizando el sintagma "educación de calidad"
}

Academic training in information and communication: problematizing the phrase "quality education"

Formação acadêmica em informação e comunicação: problematizando a frase "educação de qualidade"

\section{Yanet FUSTER CAUBET}

Universidad de la Republica - Uruguay

yanet.fuster@fic.edu.uy

Chasqui. Revista Latinoamericana de Comunicación

N. ${ }^{\circ}$ 147, agosto-noviembre 2021 (Sección Diálogo de Saberes, pp. 281-296)

ISSN 1390-1079 / e-ISSN 1390-924X

Ecuador: CIESPAL

Recibido: 02-04-2021 / Aprobado: 10-07-2021 


\title{
Resumen
}

¿Qué entendemos por educación de calidad en el contexto universitario? Este recorrido se detiene en el concepto de calidad aplicado a la educación en el nivel terciario y hace foco en un caso vinculado a la formación de los profesionales de la comunicación y la información. Este ensayo busca promover la reflexión respecto de la importancia de participar en la construcción de comunidades de discurso crítico que habiliten a los estudiantes a la formación de opinión. En las conclusiones se realizan aportes en la línea de generar consciencia sobre el compromiso social que conlleva el rol de los profesionales vinculados con estas disciplinas en la formación de una ciudadanía democrática.

Palabras clave: enseñanza desde las disciplinas, comunicación, información, prácticas letradas, Uruguay.

\begin{abstract}
What do we understand by quality education in the university context? This tour stops at the concept of quality applied to education at the tertiary level and focuses on a case related to the training of communication and information professionals. The essay seeks to promote reflection on the importance of participating in the construction of communities of critical discourse that enable students to form opinions. In the conclusions, contributions are made in the line of generating awareness about the social commitment that involves the role of professionals linked to these disciplines in the formation of a democratic citizenship.
\end{abstract}

Keywords: teaching from disciplines, communication, information, leters practices, Uruguay.

\section{Resumo}

O que entendemos por educação de qualidade no contexto universitário? Este percurso pára no conceito de qualidade aplicado à educação de nível superior e centra-se num case relacionado com a formação de profissionais de comunicação e informação. O ensaio busca promover a reflexão sobre a importância de participar da construção de comunidades discursivas críticas que possibilitem aos alunos formarem opiniões. Nas conclusões, são feitas contribuições no sentido de gerar consciência sobre o compromisso social que envolve o papel dos profissionais vinculados a essas disciplinas na formação de uma cidadania democrática.

Palavras-chave: ensino de disciplinas, comunicação, informação, práticas de letramento, Uruguai. 


\section{Introducción}

El sintagma "educación de calidad" reviste un entramado complejo, en él se interconectan una serie de descriptores que operan de forma situada, es decir, dependiendo del contexto, de los actores involucrados y de lo esperado en términos de eficacia y eficiencia, se establecerá el alcance de esa expresión. Este trabajo se detiene en el concepto de calidad aplicado a la educación en el nivel terciario y hace foco en un caso, con el objetivo de poder aplicar a una realidad concreta algunos de los planteos efectuados a lo largo del recorrido.

Me desempeño como docente universitaria en el contexto de la Facultad de Información y Comunicación (FIC), un espacio académico donde en Uruguay se imparten las licenciaturas en Bibliotecología, en Archivología y en Comunicación. Desde ese lugar me sitúo como enunciadora académica de la presente contribución, haciendo hincapié en algunos aspectos que entiendo que aportan a la calidad en la formación de los futuros profesionales de la comunicación y la información.

En los últimos años el sintagma "educación de calidad" ha sido objeto de análisis y revisión desde variadas perspectivas teóricas, y también se ha abordado desde organizaciones que se ocupan de generar condiciones para el desarrollo humano. Claro ejemplo de lo antes mencionado es la ONU, quien a través de la Agenda 2030 plantea una serie de objetivos y metas para alcanzar el desarrollo sostenible de las naciones. El objetivo 4 busca "garantizar una educación inclusiva, equitativa y de calidad y promover oportunidades de aprendizaje durante toda la vida para todos" (ONU, 2015). Cabe señalar que este objetivo está mayormente orientado hacia la enseñanza primaria, pero a los efectos de este trabajo se proyectará hacia la educación terciaria, ya que el foco de interés en este caso gira en torno a la enseñanza en contexto universitario.

Con relación al sintagma educación de calidad, antes expuesto, me pregunto: ¿Qué aspectos se deberían tomar en cuenta para brindar a los estudiantes una educación de calidad? ¿Qué concepciones docentes se espera que estén presentes en la educación terciaria? ¿Cuáles son las expectativas respecto al rol docente en este contexto? ¿Qué nivel de participación deberían tener los estudiantes universitarios en el diseño de sus propios trayectos formativos?

Estas interrogantes vertebrarán la presente contribución con el objetivo de poner en diálogo el sintagma educación de calidad con la enseñanza en información y comunicación. Sumado a ello, se espera promover la reflexión sobre la importancia de construir comunidades de discurso crítico que habiliten a los estudiantes a la formación de opinión, desde donde puedan desarrollar sus capacidades en un entorno educativo modelado a tal fin. Este punto reviste capital importancia con relación al quehacer de estas disciplinas, por el trabajo que realizan los comunicadores y los cientistas de la información, y es visto como una oportunidad para seguir avanzando en la profundización de 
una institucionalidad compartida que permita que las carreras que se imparten en la FIC interactúen aún más.

\section{Enseñanza de calidad en contexto universitario: la docencia interpelada}

En este apartado me detendré en consideraciones vinculadas con el sintagma "enseñanza de calidad". Si bien la calidad es una propiedad que reviste una valoración acerca de las cosas y por lo tanto posee connotaciones subjetivas, podemos brindar un acercamiento al punto que permita evidenciar qué se entiende por calidad en el contexto de este trabajo.

Como es notorio, se trata de un concepto situado, vale decir que no tiene una acepción estable, sino que dependerá de la situación en la que se lo aplique, del campo epistemológico que oficie como marco y de los intereses de quienes construyen la valoración. Tomando en cuenta lo expuesto, a continuación, se brindarán lineamientos que permitan conocer la concepción de calidad que se sostiene en este trabajo, en este caso con foco en la educación terciaria.

Un aspecto que se considera clave a la hora de hablar de calidad en educación en este contexto es asumir el propósito de minimizar las propuestas que conducen a la repetición de contenidos y a su reproducción. Es necesario que de forma explícita se habilite al estudiante para que tome posición sobre temas que muevan la reflexión y conduzcan a la toma de decisiones informada. La universidad tiene la responsabilidad de formar sujetos capaces de analizar y cuestionar la información a la que acceden como condición para el desarrollo humano. Si bien esto es crucial en la formación de todos los estudiantes universitarios, en el caso que nos ocupa esto adquiere especial relevancia, por la tarea que desarrollan los comunicadores y los profesionales de la información.

Desde este planteo, no entendemos la calidad en términos de un servicio que se brinda, sino con relación a los procesos y los métodos que hacen posible que los individuos adquieran herramientas que les permitan desarrollar sus capacidades para funcionar en sociedades más justas y democráticas. Sobre esto volveremos en el siguiente apartado, pues como dijimos, si bien esto debería ser parte de la formación de cualquier estudiante, sobre todo en el ámbito de formación terciaria, se trata de aspectos muy sensibles a la hora de pensar en los futuros profesionales de la información y la comunicación.

Los docentes tenemos la responsabilidad de acompañar y habilitar a los estudiantes para que aprendan a funcionar en este nivel de formación, ayudándolos a realizar un examen activo sobre el conocimiento al que acceden, promoviendo el análisis de evidencias o razones que les permitan decidir y alentar la búsqueda de elementos necesarios para formar opinión. Un camino para lograrlo puede ser el uso de estrategias metacognitivas para el aprendizaje.

La metacognición puede concebirse como la conciencia acerca del propio conocimiento, y por ende involucra la posibilidad de potenciar esa capacidad 
trabajando deliberadamente la atención y la comprensión. Los docentes devienen en pieza clave de este proceso, pues son quienes habilitan (o no) el vínculo del estudiante con su propio aprendizaje.

El enfoque metacognitivo permite dotar de calidad a la educación, desde la perspectiva de los docentes, pues es una estrategia de enseñanza, con relación a los estudiantes la autorregulación del propio aprendizaje opera como agente motivador, al dar autonomía al estudiante, a la vez que desarrolla la capacidad de expresarse con libertad, focalizando en potenciar las posibilidades de expresar su pensamiento a través de la palabra.

La autonomía intelectual es una capacidad que no se desarrolla naturalmente, por el mero hecho de ingresar en un ámbito de educación terciaria, por eso los docentes deberíamos acompañar su cultivo. En tal sentido, es necesario planificar estrategias de enseñanza a través de las cuales se puedan ir generando las condiciones de producción de los saberes, reconociendo el éxito en los aprendizajes y alentando sobre las oportunidades de mejora. Favorecer el desarrollo del pensamiento crítico se entiende clave si pretendemos brindar una enseñanza de calidad y como habilidad metacognitiva integra la concepción de enseñanza de calidad que se plantea en este artículo.

Lo expuesto está estrechamente vinculado con las condiciones de producción y circulación de los discursos, por ende la lectura y la escritura tienen un lugar clave. Si bien esto es notorio, y puede resultar una obviedad, los procesos de alfabetización académica deben ser explicitados, pues a lo largo de la vida universitaria la lectura y la escritura también se aprenden. Es responsabilidad de la universidad asumir su enseñanza y desarrollar una comunidad de lectores y escritores donde los contenidos disciplinares se aprendan a través de prácticas letradas situadas, vale decir, en consonancia con los requerimientos de las disciplinas.

Con relación a las disciplinas vinculadas con la información y la comunicación, la lectura y la escritura revisten un papel clave, porque además de vehiculizar el conocimiento que adquirirán a lo largo de la carrera, desde donde también adquirirán herramientas para producir textos, estas prácticas ocuparán un espacio medular en su vida profesional, pues se trata de campos del conocimiento que realizan una especial contribución a la transformación social, sin dudas que cultivar la mirada crítica sobe lo que se lee y sobre lo que se escribe es condición sine qua non para formar futuros profesionales de la información y la comunicación.

Con el objetivo de situar a la enseñanza de calidad con foco en lo disciplinar, en el siguiente apartado nos detendremos en algunos aspectos que ilustran el trayecto que se ha venido transitando en la FIC 


\section{La Licenciatura en Comunicación y la Licenciatura en Información en Uruguay: en búsqueda de una institucionalidad compartida}

Tal como mencionara anteriormente, en la FIC se cursan las licenciaturas en Bibliotecología, Archivología y Comunicación. La integración de las tres carreras en una misma facultad es reciente, data de 2013, cuando el Consejo Directivo Central de la Universidad de la República (Uruguay) aprobó su creación. Antes de ello funcionaban en servicios universitarios separados, por un lado, Archivología y Bibliotecología se dictaban en la Escuela Universitaria de Bibliotecología y Ciencias Afines (EUBCA) y Comunicación en la Licenciatura en Ciencias de la Comunicación (Liccom). La razón de la integración radica en la constatación de que existen zonas disciplinares comunes entre las carreras. La sociedad actual es claro ejemplo de la complejización de los fenómenos que involucran a la información y a la comunicación. La multiplicación de fuentes y recursos de información, y sus mecanismos de difusión, sumado a la diversidad de prácticas sociales y discursivas vinculantes en clave de información y comunicación, dan evidencia de la potencialidad y proyección futura que tienen estas carreras, y de la necesidad de pensarlas de manera integral. Los destinos de la sociedad en su conjunto cada vez más se decidirán en los campos disciplinares antes mencionados.

En este contexto, los docentes debemos promover el desarrollo de habilidades de comprensión y análisis que le permitan a los estudiantes interpretar la información para poder gestionarla adecuadamente y promover su recuperación, aspectos que transversalizan la propuesta de enseñanza en las licenciaturas en Archivología y Bibliotecología, así como también generar en los futuros comunicadores la conciencia acerca de la responsabilidad de su rol en la construcción de una ciudadanía capaz de formar opinión sobre los temas que impactan en la sociedad, para poder tomar decisiones informadas. El desarrollo del sentido crítico y la capacidad de discernimiento nos harán ciudadanos más responsables y cultos, y también profesionales competentes en relación con los requerimientos sociales.

La construcción de abordajes situados, en consonancia con los trayectos curriculares propuestos en los planes de estudio, y pensados desde la experiencia en las aulas, se presenta como una oportunidad para que los docentes intervengan adaptando ideas y prácticas de enseñanza ya implementadas y que resultaron exitosas, a la vez que encuentren vías para socializar sus propias prácticas para que otros puedan replicarlas.

Una vía posible puede ser la construcción de abordajes compartidos sobre el fenómeno de la información y su comunicación que desde una postura crítica integren al discurso el espacio crucial que estas disciplinas ocupan con relación a las personas y sus necesidades. 
Desde este lugar las prácticas de enseñanza son interpeladas, pues la horizontalidad en el diálogo con los estudiantes y la apertura a nuevas posibilidades de desarrollo del proceso educativo se plantean como concepciones docentes que es necesario sostener en el trabajo de aula. En el ámbito universitario, los docentes tienen la responsabilidad de desarrollar acciones que acompañen a los estudiantes para que puedan insertarse en la cultura discursiva de las disciplinas. Un camino posible podría estar vinculado con el tratamiento de los textos que componen la bibliografía recomendada en los cursos, los cuales "deberían ser sometidos a estudio y discusión desde el espacio de aula, de forma explícita, y no solo como camino a recorrer por los estudiantes fuera del aula para complementar lo expuesto en clase" (Fuster, 2016, p. 8).

No parece suficiente proponer temas que acompañen el devenir de los tiempos, también la metodología de trabajo debe caminar en consonancia con el desarrollo de una postura crítica, intentando que el estudiante se nutra de las instancias del proceso de enseñanza de forma activa, y sintiéndose habilitado a realizar propuestas. Para lograrlo es clave que los estudiantes se empoderen de su propio trayecto de formación e intervengan, aportando desde su rol a la construcción del camino universitario. En Uruguay, el plan de estudios vigente para estas carreras (Plan de estudios 2012) habilita a llevar adelante estas acciones, pues ofrece trayectos abiertos para que los estudiantes puedan ir tomando decisiones y efectuando sus propias elecciones para cumplir con los créditos.

\subsection{Sensibilidad y responsabilidad en la formación de los futuros profesionales de la comunicación y la información}

Es clave asumir que las transformaciones que se operan en las sociedades no necesariamente implican avances. Exclusión, lejanía e inequidad caminan paradójicamente junto al desarrollo, en un mundo cada vez más globalizado, donde la brecha informacional que separa a los inforricos y a los desvalidos informacionales es cada vez más profunda.

Sería oportuno abordar tópicos como los mencionados desde las prácticas letradas que se desarrollan en la formación de los futuros profesionales de la comunicación y la información. Concebir las actividades de lectura y escritura como prácticas letradas implica asumir que cada palabra elegida, cada término empleado a la hora de producir un texto e incluso la organización que se le da habla del momento socio-histórico y cultural y del devenir de quienes participan en esa comunidad que lo ha producido. Las prácticas letradas "consisten en los comportamientos y conceptualizaciones social y culturalmente compartidos que dan sentido a los usos de leer y escribir en diferentes situaciones" (Orlando, 2013, p. 70). 
Este planteo, llevado a la enseñanza de la información y la comunicación, podría ser transversal a las distintas áreas del conocimiento que componen la malla curricular de las carreras, pues se trata de prácticas que buscan que el estudiante sea capaz de formar opinión y de reflexionar a partir de las lecturas que realiza. Para acompañar esta concepción el docente tendría que habilitar desde lo metodológico y lo conceptual, y también desde las concepciones referidas a la evaluación, los caminos para que el estudiante desarrolle opinión sobre lo que lee y sobre los procesos de escritura que devienen en el ámbito académico.

La ciencia de la comunicación y la ciencia de la información son ciencias sociales, y como tales disciplinas cuyo estilo discursivo tiene un componente fuerte de exposición y argumentación. El empleo de vocabulario técnico y de conceptos complejos exige lectores con un bagaje cultural que les permita realizar un análisis que trascienda lo que está dicho de forma explícita. Adherimos a las palabras de Shera (1990, p. 36), sin dudas aplican en relación con los licenciados en información y también en comunicación: "la educación del bibliotecario debe abarcar mucho más que una pericia técnica. Nadie puede negar que el bibliotecario debe 'saber sobre libros', pero debe saber mucho más que eso, en el más amplio y rico sentido debe ser una persona instruida”. Promover estrategias que atiendan la lectura desde una postura crítica puede favorecer, por un lado, a profundizar la comprensión de lo leído, y por otro, a trabajar la información y su comunicación como objeto de estudio desde una mirada que habilite nuevas lecturas, en plural.

Desde este enfoque, promover la enseñanza de textos por donde circula el discurso especializado implica habilitar al estudiante para que identifique los conceptos vertebradores del texto y sea capaz de sostener un planteo propio, identificando el género discursivo más adecuado en cada caso para plasmar sus ideas en el instrumento comunicativo que es el texto académico. Esto lo ayudará a iniciarse como productor de textos dentro de esa comunidad discursiva en la cual está desarrollando su trayecto de formación (Fuster, 2011). De esta forma, la formación que el estudiante adquiera le permitirá cimentar las bases de lo que será como profesional, no como repetidor de los saberes con los que fue tomando contacto en la carrera, sino como hacedor de nuevas miradas que a su vez permitan generar polifonía con otras voces que ensanchen el espectro de lo conocido. Esto aporta a los actores sociales otras formas de observar el mundo que le permitirán estar preparados para nuevos retos.

El desarrollo de prácticas discursivas que promuevan el sentido crítico y que se tomen como transversales en las carreras podría ser una vía de interacción entre los docentes abonando al sintagma "educación de calidad" en el nivel terciario. Favorecer la construcción de comunidades de discurso crítico que impulsen a los estudiantes a la toma de decisiones e intervención práctica en contextos donde puedan aplicar las competencias incorporadas en un entorno educativo modelado a tal fin, se presenta como una vía de intervención sobre el 
punto. En tal sentido creo viable poner en práctica la organización curricular flexible promovida desde los documentos institucionales (plan de estudios y otras disposiciones que regulan los procesos de enseñanza), sin desatender el rigor académico.

La organización curricular flexible habilita al estudiante a ser partícipe de su propio proceso, brindando opciones para que este pueda orientar su trayecto en función de sus intereses, lo que opera como un agente motivador muy potente. La flexibilidad en el currículo rinde en la medida en que exista una oferta amplia de cursos que contemplen perfiles estudiantiles variados, solo desde ese lugar el estudiante podrá desarrollar genuinamente la capacidad de elección y la toma de decisiones, aspectos que lo preparan más allá de lo académico, ya que son herramientas para la vida. El entorno educativo debe estar modelado atendiendo estos aspectos, y el docente deberá concebir sus estrategias de enseñanza, sus métodos e instrumentos de evaluación en esta línea, pues es quien deberá observar los trayectos desarrollados por los estudiantes, y direccionar las acciones para que el estudiante aprenda a aprender.

\subsubsection{Experiencias de enseñanza desde las disciplinas en la FIC}

En la FIC se llevan a cabo estrategias de enseñanza en clave de diálogo disciplinar entre la comunicación y la información, por eso a continuación me detendré es este caso y compartiré una experiencia que tal vez pueda ser aplicada a otros contextos, con los debidos ajustes que cada situación amerite. Si bien el hecho de centrarnos en un caso focaliza en una particularidad, podemos hablar de transferibilidad y no de generalización a la hora de socializar experiencias como la que mencionaré a continuación y desde ese lugar la presento, con el propósito de brindar aportes que puedan dar sustento a una enseñanza de calidad en el nivel terciario.

Es necesario plantear que esta no es la única experiencia donde la enseñanza de las disciplinas es abordada en el contexto de la FIC, pero me interesa detenerme en esta en particular pues se trata de un abordaje que se propone atender de forma deliberada la lectura y la escritura de los estudiantes y el desarrollo del pensamiento crítico, aspectos que como vimos en este recorrido se entienden como cruciales en la formación de los profesionales de la comunicación y la información.

En este caso referiré al trabajo que desde el año 2019 viene realizando un grupo de docentes (pre)ocupados por la lengua escrita. El mencionado grupo presentó una propuesta de trabajo titulada "Formación docente para promover la mejora del manejo estudiantil de la lengua oral y escrita. Cursos y niveles en la FIC”. El propósito que los guía es compartir materiales y actividades pensados para el apoyo al trabajo con la lectura y la escritura dentro de los diversos cursos que se dictan en la mencionada facultad, partiendo de la idea de que podemos tener estudiantes aún mejores y que: 
1. A leer y a escribir se aprende también a lo largo de la vida universitaria.

2. No hay lectura ni escritura sin contenidos disciplinares.

3. La FIC es responsable de nutrir estos procesos de aprendizaje de la lectura y de la escritura académicas.

4. Los docentes, a veces, no tenemos en claro qué pueden hacer los estudiantes y, en consecuencia, qué precisan de nosotros.

5. Los docentes, como modelos de lectores y escritores para los estudiantes, debemos hacer explícitas nuestras prácticas para tener una comunidad lectora y escritora más sólida.

6. La FIC debe ser un entorno académico confortable en el que habiten estudiantes responsables y que sepan que aprender es tan disfrutable como trabajoso (LesFIC, 2020). ${ }^{1}$

Los presupuestos teórico-metodológicos que sustentan las actividades del grupo LesFIC van en sintonía con la intención de promover una enseñanza de calidad en el contexto universitario, ya que no focalizan en la lectura y la escritura como adquisición de habilidades (adquiridas de una vez y para siempre, separadas del contexto en que circulan los discursos), sino como prácticas que entienden al lenguaje como fenómeno social. En estos campos disciplinares la información no solo es vehículo para aprender, sino que además es objeto de estudio al que se accede a través de prácticas letradas. Las propuestas del grupo son variadas, a través de ellas se proponen distintas formas de leer y aproximarse a la información, distintos acercamientos para comprender lo leído y también para producir conocimiento. Es precisamente sobre esta diversidad que se articulan los aprendizajes y entiendo que esta es una oportunidad de trabajo coordinado entre los equipos docentes, con el objetivo de promover la educación lingüística aplicada a la enseñanza de las disciplinas.

Es necesario señalar que para elaborar el presente artículo no se realizó una investigación que brindara datos que permitan conocer de qué forma las propuestas antes mencionadas han impactado en las prácticas de enseñanza de la FIC. Tampoco está relevada la existencia de trabajo compartido entre equipos docentes que brinden cursos en comunicación e información, lo que ayudaría a conocer su incidencia en la construcción de una cultura institucional que atienda este diálogo disciplinar. La mención a este caso se realiza con la finalidad de situar el planteo que vertebra este texto sobre el sintagma educación de calidad, y presentar una posible ruta de trabajo compartido que a su vez se pueda extrapolar a otros contextos de enseñanza de estas disciplinas.

1 Para ampliar esta información se sugiere visitar el sitio de LesFIC, disponible en https://les.fic.edu.uy/, allî podrán encontrar propuestas de actividades para realizar con los estudiantes. Todas ellas están pensadas con relación a la formación de los futuros licenciados en Archivología, Bibliotecología y Comunicación, y ofrecen lineamientos que permiten adaptarlas a las realidades concretas del trabajo en el aula desde determinada asignatura. 


\subsubsection{La enseñanza de calidad debe estar atenta a los cambios}

Otro aspecto que es interesante atender tiene que ver con los cambios que las disciplinas en sí mismas han experimentado a lo largo del tiempo. Respecto a la Archivología y la Bibliotecología debería pensarse el desempeño del profesional de la información trascendiendo el rol tradicional vinculado al trabajo en el archivo o en la biblioteca, y desterrar del imaginario el énfasis que se ha puesto en el espacio físico, como si el archivo o la biblioteca fueran determinantes por sí solos de la actividad que desarrolla el profesional de la información. Es necesario habilitar instancias que permitan pensar en el trabajo con la información más allá del lugar que da nombre a la profesión. Es oportuno plantear además que sería oportuno pensar también en un cambio de óptica para entender "la biblioteca”, y verla más allá de sus anaqueles cargados de libros, concibiéndola como un espacio comprometido de resistencia cultural, identitaria y colectiva.

Para que esto sea posible es necesario generar conciencia del rol social que emerge y constituye una nueva forma de pensar el trabajo de los bibliotecólogos, más adecuado a los actuales tiempos de infoxicación y sobreabundancia de información vehiculizada a través de diversos soportes. Este es un componente del ejercicio de la disciplina que debería integrarse de forma más potente a la enseñanza en ciencia de la información y desde el cual se pueden trazar puntos de convergencia con el rol del profesional de la comunicación, el cual también ha ido virando hacia nuevas formas de entender el ejercicio de la profesión. La participación sostenida de los profesionales de la comunicación y de la información en las políticas públicas también es un signo de estos tiempos que debe construirse desde la formación, para continuar trabajando en la apertura de instancias de debate que confluyan en un diálogo que apueste por ejemplo a una comunicación alternativa ${ }^{2}$ (Kaplún, 2013; 2019), a una bibliotecología social (Parada, 1999) y a una bibliotecología progresista (Civallero, 2016), ya que estas propuestas invitan a una mirada crítica y reflexiva sobre la realidad.

El bibliotecario debe aprender (o recordar) el significado de las expresiones "responsabilidad social", "ética profesional" y "compromiso". No son términos vacíos: son locuciones cargadas de valor, nacidas al amparo de unos ideales y de unas creencias firmes en la igualdad de oportunidades, los derechos humanos, la libertad y la solidaridad. El bibliotecario, la biblioteca y su entorno son parte de un todo que está sujeto a los problemas que golpean a la sociedad en general. (Civalero, 2016)

Concebida como un espacio comunitario, la biblioteca tiene la misión de atender las necesidades de los usuarios, brindar servicios para todos y ampliar

2 Si se desea ampliar respecto a los orígenes de estos movimientos que buscan miradas alternativas a las dominantes en nuestras sociedades y que hacen foco en los desafíos que plantea la lucha por el derecho a la comunicación en los contextos actuales, sugiero consultar a Kaplún (2007; 2019), Kejval (2019) y Lemus Pool (2017) 
los canales de comunicación apostando a la cohesión social, como plantean las corrientes sociales vinculadas a la bibliotecología. De acuerdo a los planteos que emergen desde la bibliotecología progresista, los profesionales tienen el compromiso de trabajar para torcer el curso de las corrientes de exclusión que amplían la brecha entre los que acceden a la información y los desvalidos informacionales. Siguiendo esta línea se trabaja por la descolonización del saber, construyendo comunidades que producen el saber desde sí mismas, situadas desde su propio discurso (Lopera, 2006).

En el ámbito de la comunicación también se han ampliado los espacios para dar cabida a otras voces, lo que permite ensanchar el espectro de opciones para que los sujetos sociales participen y polemicen acerca de las variadas formas de leer y entender los medios y la comunicación en el entramado social y cultural de nuestro tiempo. Simpson Grinberg (1989, p. 41) define lo alternativo como "todo medio que, en un contexto caracterizado por la existencia de sectores privilegiados que detentan el poder político, económico y cultural [...] implique una opción frente al discurso dominante". Si bien coincido con Kaplún (2019, p. 73) en que "lo alternativo" es una categoría relativa, pues se construye como un otro frente a lo hegemónico, que se le opone o que lo complementa, tanto la bibliotecología social como la comunicación alternativa giran en torno a un proyecto de cambio profundo en la sociedad y suponen una crítica al colonialismo cultural caracterizado por la mirada que cuestiona y que busca otras epistemologías para entender los fenómenos sociales.

Estos planteos evidencian la polifonía de voces que van marcando su impronta, a la que se le suman aportes que, desde distintos campos, no solo desde la academia sino también desde otras trincheras aportan al diálogo comunicación/información-sociedad. En esta línea, y con relación a comunicación, Kaplún (2019, p. 83) plantea: "nuestro campo de estudio y acción nos obliga hoy a tejer más interdisciplinariamente. Y a indisciplinar aún más nuestro oficio, en imprescindible diálogo de saberes con otros que están fuera y lejos de la academia, pero tienen mucho para aportar a la comprensión y construcción de una comunicación alternativa. O muchas". Como esferas de la actividad humana, las disciplinas circulan a través de discursos, y es desde ellos que vamos comprendiendo la lógica de funcionamiento que las vertebra y los caminos que las acercan a la praxis, caminos diversos que invitan a transitarlos. Es necesario que estas cuestiones se integren a la enseñanza de las disciplinas y se habilite la participación.

\section{Consideraciones finales}

A lo largo de este recorrido focalicé en la importancia de atender los puntos de convergencia que poseen la comunicación y la información como disciplinas, en aras de profundizar aún más los lazos que permitan seguir construyendo una institucionalidad compartida en el contexto de la FIC. Como una estrategia 
posible de vinculación disciplinar, esta contribución se detiene en el valor del análisis crítico como metodología de abordaje de los textos, de forma transversal a la formación de los profesionales de la información y la comunicación.

Una enseñanza de calidad concibe al conocimiento no como un fin, sino como un medio de aproximarnos y comprender nuestra circunstancia y el mundo en el que estamos inmersos, y desde ese lugar posicionar nuestra voz. "Esto implica la construcción de una sociedad integrada por seres responsables de sus ideas, que no solo encuentran vías de acceso a la información, sino que a partir de ellas logran empoderarse del discurso, apostando fuertemente a la formación de una ciudadanía democrática" (Fuster, 2011).

He planteado el caso de la FIC por ser el entorno institucional donde desarrollo mi práctica docente en Uruguay, y desde donde intento instalar prácticas de lectura y escritura que promuevan el sentido crítico. Más allá de eso considero que los planteos desarrollados en este recorrido bien pueden extrapolarse y situarse, con los ajustes necesarios, a otros contextos. Debido a esto he entendido oportuno compartir estas disquisiciones con el objetivo de poner sobre la mesa el tema de la enseñanza de las disciplinas, y el valor dado a la lectura y a la escritura en el contexto universitario, con relación al sintagma "educación de calidad".

Creo oportuno sumar a estas consideraciones finales la siguiente interrogante: ¿basta con poseer una conciencia crítica? Opino que de poco sirve una conciencia crítica que no se vincule con la acción colectiva, y por esa razón tal vez lo que necesitamos son conciencias críticas que se desarrollen en función de situaciones concretas y que actúen en contextos que permitan a la gente informarse para la toma de decisiones, y conocer múltiples miradas sobre los fenómenos que impactan en la sociedad.

Sensibilidad y responsabilidad son expresiones que transversalizan una propuesta de enseñanza de calidad en los actuales tiempos, y son conceptos que se deben aplicar tanto a escala estudiantil como desde las concepciones docentes. Los docentes deben asumir esta responsabilidad pues sus aportes son claves en la formación de los futuros profesionales. Cada vez es más fuerte la necesidad de educar para el cambio social y hacer foco en los estudiantes, no solo en sus dificultades, sino especialmente con relación a lo que son capaces de hacer.

Más allá de lo expuesto, es necesario destacar que formar en pensamiento crítico no interpela solo al rol docente, sino que es clave que se conciba desde una perspectiva institucional, para que no se transforme en intentos aislados de algunos docentes y que el estudiante experimente un hiato respecto a lo que se espera de su participación en el proceso de enseñanza-aprendizaje del cual es protagonista.

¿Qué caminos pueden seguir los estudiantes para demostrar su compromiso con la realidad, con su transformación y con el uso de herramientas de comunicación? Se necesitan estudiantes comprometidos, 
solo de esta forma podrán involucrarse y sentir que tienen en sus manos la inmensa responsabilidad de informar, y de hacer llegar a las comunidades las herramientas que les permitan formar opinión sobre la información que reciben. Cuando los individuos o grupos se comprometen, participan de la transformación de la realidad y sienten que tienen parte en la construcción de un mundo menos individualista, más solidario y empático. Los profesionales de la comunicación y de la información son mediadores, llevan el conocimiento a la sociedad, y asisten desde su expertisse para que los saberes circulen. Ese es el deber ético y profesional que están llamados a honrar, y es el deber de los docentes hacerlo explícito a través de la formación en el ámbito universitario, asistiéndoos para que construyan vías de actuación que se traduzcan en acción, más allá de cualquier ideología.

Como ya hemos planteado, sería deseable que estas disquisiciones puedan extrapolarse a otras realidades y entornos de formación, ya que la mirada crítica sobre lo que se lee y sobre lo que se produce no debería ser patrimonio exclusivo de las carreras universitarias vinculadas de forma más cercana con las letras. Todos los estudiantes/futuros profesionales, en tanto sujetos inmersos en entornos sociales tienen la responsabilidad de estar informados, y poder opinar sobre los temas que les atañen como ciudadanos.

Las interrogantes planteadas al inicio de este recorrido quedan abiertas, no hay respuesta definitiva. La intención ha sido reflexionar sobre estos puntos y ver en qué medida esa reflexión puede impulsar a la acción.

\section{Referencias bibliográficas}

Civalero, E. (2016). Sobre inclusiones, exclusiones y bibliotecarios en el filo de la navaja. Revista Clip, 62. Recuperado de https://clip.sedic.es/article/inclusiones-exclusiones-bibliotecarios-filo-la-navaja/

Fuster, Y. (2011). Sociedad de la Información y literacidad crítica, implicancias en la formación del profesional de la información. En Informatio 14/16. Disponible en: http:// informatio.eubca.edu.uy/ojs/index.php/Infor/article/view/97/161

Fuster, Y. (2016). El texto académico como género discursivo y su enseñanza en la educación terciaria. En Palabra Clave (La Plata), vol. 5 (2) eoo7. http://www.palabraclave.fahce. unlp.edu.ar

Kaplún, G. (2013). Viejas y nuevas tradiciones en la comunicación latinoamericana. Revista Latinoamericana de Ciencias de la Comunicación, 10, (18), p. 66-76. Sao Paulo, Alaic.

Kaplún, G. (2019). La comunicación alternativa entre lo digital y lo decolonial. Chasqui. Revista latinoamericana de Comunicación, 141, p. 75-94, 2019.

Lopera, H. (2006). Los Estudios Culturales como alternativa para la construcción de una Bibliotecología de la esperanza. Otra bibliotecología es posible (blog). Recuperado de http://otrabibliotecologiaesposible.blogspot.com/

Organización de las Naciones Unidas (ONU) (2015). Objetivos de desarrollo sostenible. Recuperado de https://www.un.org/sustainabledevelopment/es/objetivos-de-desarrollo-sostenible/ 
Orlando, V. (2013). Sobre prácticas letradas y estudios univesitarios. InterCambios, 1, (2), p. 69-72.

Parada, A. (1999). Bibliotecología y responsabilidad social. Información, cultura y sociedad, 1 pp.65-75. Recuperado de http://eprints.rclis.org/16980/1/no1ao4.pdf

Shera, H. (1990). Los fundamentos de la educación bibliotecológica. Ciudad de México: UNAM/ CUIB.

Simpson Grinberg, M. (1989). Comunicación alternativa: Tendencias de la investigación en América Latina y Comunicación alternativa: Dimensiones, límites, posibilidades. Comunicación alternativa y cambio social. Ciudad de México: Premia Editora.

Verón, Esteban (1987). La palabra adversativa. En Verón, E., El discurso político, varios autores, (p. 13-26). Buenos Aires: Hachette. 
